\title{
A novel group decision making method to overcome the Web 2.0 challenges
}

\author{
Juan Antonio Morente-Molinera*, Ignacio Javier Pérez ${ }^{\dagger}$, Francisco Chiclana ${ }^{\ddagger}$, Enrique Herrera-Viedma* \\ * Department of Computer Science and Artificial Intelligence \\ University of Granada, Granada \{jamoren, viedma\}@decsai.ugr.es \\ †Department of Computer Science Engineering \\ University of Cádiz, Cádiz, Spain \\ ignaciojavier.perez@uca.es \\ $\ddagger$ Centre for Computational Intelligence \\ School of Computer Science and Informatics \\ De Montfort University, Leicester, UK \\ chiclana@dmu.ac.uk
}

\begin{abstract}
With the appearance of Web 2.0 technologies, the way Internet is conceived has dramatically changed. Internet users have begun to perform a more active role in providing and sharing the information available on the Web. Thanks to mobile technologies, users can access the Internet from their smartphones at any time, independently of their location. Moreover, users gather in communities where they can communicate and share information. These communities can hold a high number of people and, consequently, a large amount of information need to be managed. Nevertheless, as the information tends to be disorganized, it is difficult for users to manage them properly to make the most of it. Therefore, there is a necessity of tools that can help users to organize and manage in a proper way the large amount of available information. In this paper, we propose the use of a novel group decision making approach to allow a high number of people to communicate among themselves and reach conclusions in a regulated way. As a high amount of people usually implies too much information, we propose the use of Fuzzy Ontologies as a way to deal with it in an organized way.
\end{abstract}

Index Terms-Decision Making; Knowledge Management; Semantic Web; Web 2.0; Knowledge Representation; Fuzzy Ontologies

\section{INTRODUCTION}

Traditionally, the internet main purpose has been the search of information. Therefore, most of the users were not allowed to alter or include any piece of information, not even provide suggestions. However, since the appearance of Web 2.0 technologies [1], the above scenario has changed dramatically. In the new Internet paradigm that Web 2.0 technologies have promoted, users hold the main role. They have become the sources and consultants of all the information available on the Internet, that is, they provide and consume information at the same time. Web 2.0 technologies have contributed to the exponential increase of information that is available on the Internet and it has allowed the creation of Web applications where users can cooperate actively. Examples of these are Twitter, Facebook, Wikipedia and Ebay.

With the appearance of 3G/4G technologies [2] and smartphones [3], the necessity of Internet access points have disappeared. Therefore, Internet has become even more acces- sible. Thanks to smartphones, everyone is able to retrieve information from anywhere at any time, that is, space and time restrictions have completely disappeared. Furthermore, the appearance of Android [4] and IOS [5] operative systems have motivated the implementation of applications that benefit from the Internet stored information to help smartphones users. It is safe to say that smartphones are becoming the assistance devices that everyone needs for their everyday life.

This new environment needs tools to help users to share information, debate and make group decisions efficiently in an organized way. Although Group Decision Making (GDM) is a traditional field reported in the literature since the late 70's [6], the designed methods can easily benefit from Web 2.0 technologies in order to carry out GDM processes through the Web.

This change of paradigm, though, presents several issues that must be addressed in order to get the most out of Web 2.0 technologies. In this paper, we focus on proposing solutions for two of the main Web 2.0 challenges:

1) High number of available users: Web 2.0 allows users located everywhere in the world to share information and establish contact as if they were nearby using Internet communities. This situation does not cause any problem if the number of users that are communicating is relatively low. However, when the number of users involved is high, it is difficult for them to communicate efficiently, make decisions and reach common goals without any assistance. Therefore, in this case, there is a need of tools to support users to discuss matters in an organized way using Internet and Web 2.0 technologies. Moreover, when a large set of experts are involved in a decision making process and they use Internet as a platform for communicating, it is common that they are not continuously online. Therefore, they usually leave the decision process and rejoin at a later time. In the literature, Alonso et al. [8] have already proposed a GDM method that is able to tackle the problems related 
with this specific Web 2.0 issue by incorporating a new delegation scheme that allows experts to delegate in others that represent their opinions.

2) Large amount of available information: The high number of users surfing on the Internet has provoked an exponential increase of the available information. Tools able to deal with environments that hold a high number of users proposed in [8] are no solution to the large amount of information issue. To deal with this second challenge, we propose the use of Fuzzy Ontologies as data storage and management tool.

In this contribution, we propose a new extension of the GDM method proposed in [8] that is capable of dealing with both Web 2.0 issues described above. For this purpose, a delegation scheme is used to deal with the high number of available users in a Web community, which makes use of Fuzzy Ontologies to deal, store and organize the high amount of available information.

In Section II, the basis of both GDM and Fuzzy Ontologies are provided. In Section III, the novel designed method is exposed. In Section IV, advantages and drawbacks of the proposed solutions are discussed. Finally, in Section V some conclusions are drawn.

\section{PReliminaries}

In this section, GDM and Fuzzy Ontologies basis are exposed.

\section{A. Group Decision Making}

Day-to-day, people have to make decisions. Most of them are made by a set of individuals. In order to assist them to select the right choice, GDM methods are developed. Formally, a GDM problem can defined as follows [9]:

Definition 1. Let $E=\left\{e_{1}, \ldots, e_{m}\right\}$ be a set of experts that must make a decision and let $X=\left\{x_{1}, \ldots, x_{n}\right\}$ be a set of alternatives from which the best one is to be chosen. A GDM problem aims to produce a ranking of the elements of set $X$ using the set of preferences preference values $P(X)$ provided by the set of experts $E$.

In GDM methods, there are usually two separated processes that are carried out to reach a final decision result [10]:

- Consensus process: The consensus process tries to help experts to reach a consensual decision. It is important to allow experts to debate, share ideas, and help them to make a unanimous decision. The more consensual a decision is, the more experts agree with it meaning that a long debate has been carried out, otherwise the decision is trivial. It is important to make decisions carrying out a debate in which all the advantages and drawbacks of the alternatives are exposed and help experts to avoid making careless decisions. In the literature, there is a high number of GDM methods that include consensus measures to promote debate [11], [12]. These methods measure the consensus achieved in each GDM round and promote debate when consensus is not achieved.

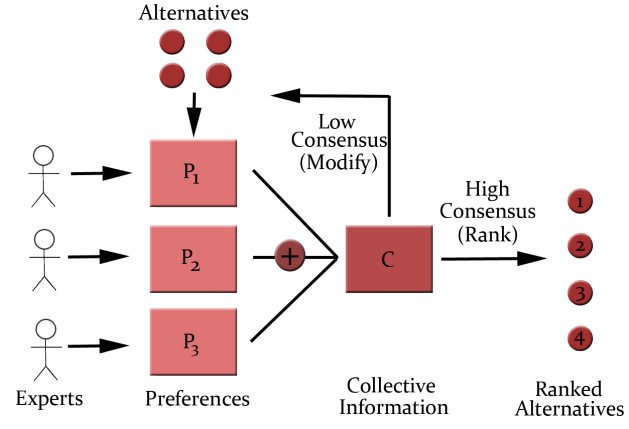

Fig. 1. Graphical scheme of a GDM process.

- Selection process: When consensus measures stablish that a high percentage of experts agree with the decision making results, the alternatives ranking is produced. The decision making result is considered optimal since most of the experts agree.

Generally, to solve a GDM process that implements consensus measures, the following steps are completed:

1) Preferences provision: Experts provide their preferences on the set of alternatives. There are several structures that experts can select. Some of them are described in [13].

2) Collective value calculation: Experts' preferences are aggregated into a single collective preference representing the overall opinion of all the experts.

3) Consensus measures calculation: Using the individuals preferences values, consensus measures are computed to determine levels of disagreement. If the level of agreement is low, experts are asked to debate and change their preferences in order to reach a consensus. It is possible to use feedback processes [14] to suggest experts how to modify their preferences.

4) Ranking calculation: When the consensus is high or a specific number of decision making rounds has been reached, the selection process is applied, alternatives ranking is obtained and the GDM process is ended.

Figure 1 shows a graphical scheme of the described process.

\section{B. Fuzzy Ontologies}

Ontologies [15] are tools able to store information in an organized way that allow users to manage and analyse. An ontology is defined as a quadruple $(I, C, R, A)$ formed by the following elements:

- Individuals $(I)$ : They are entities that can be described using concepts.

- Concepts $(C)$ : They are perceptions used to associate descriptions to the individuals stored in the ontology.

- Relations $(R)$ : They are used to create relationships among individuals and individuals and concepts.

- Axioms $(A)$ : They are rules that are fulfilled by the elements of the ontology.

In the beginning, ontologies were based on description logics. This fact makes them unsuitable for dealing with impre- 


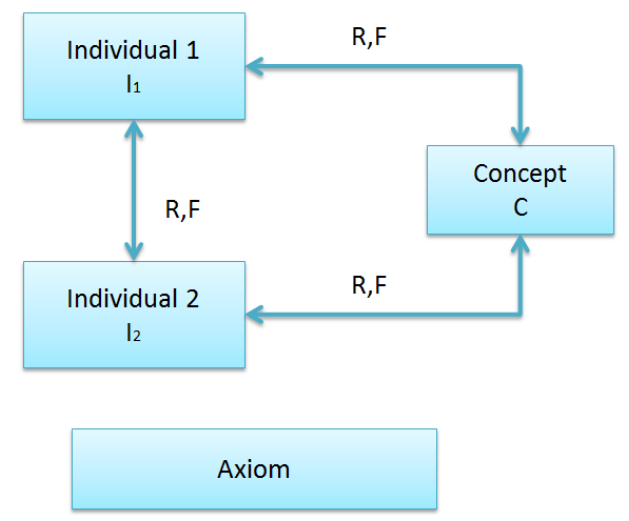

Fig. 2. Fuzzy Ontology scheme.

cise information [16]. In order to make ontologies compatible with fuzzy nature elements such as linguistic labels, Fuzzy Ontologies were developed. Bobillo et al. [17] provides the following definition of Fuzzy Ontology: "A Fuzzy Ontology is simply an ontology which uses fuzzy logic to provide a natural representation of imprecise and vague knowledge and eases reasoning over it". One of the most used ways of defining Fuzzy Ontologies is exposed below:

A Fuzzy Ontology [18], [19] can be defined as a quintuple $O_{F}=(I, C, R, F, A)$ where $I$ is a set of individuals, $C$ is a set of concepts, $R$ is a set of relations, $F$ is a set of fuzzy relations and $A$ is the set of axioms. A graphical scheme of a Fuzzy Ontology is shown in Figure 2.

The $F$ element allows the definition of fuzzy relations. Thanks to them, individuals can be related to concepts to an specific degree. When using $R$, each individual is totally related with each concept or not related at all, that is, the only degrees used are $\{0,1\} . F$ allows each individual to be related with each concept using a membership degree that is usually defined over the interval $[0,1]$. The mathematical environment of fuzzy sets [20] is used to define this relation.

Fuzzy Ontologies topic is widely used in recent literature. For instance, in [21] Fuzzy Ontologies are used to model and recognize human behaviour. In [22], Fuzzy Ontologies are used to create an intelligent system that help to determine the level of severity and treatment recommendation for Benign Prostatic Hyperplasia disease. Not surprisingly, Fuzzy Ontologies have been recently used in the field of group decision making [23].

\section{A NOVEL GRoup DeCision MAKING METHOD FOR WEB 2.0 ENVIRONMENTS}

In this section, we present the new GDM method that has been specifically designed for working on Web 2.0 environments with a high number of users and large amount of information.

\section{A. Integrated novelties of the proposed GDM process}

The main highlights of the method are based on adding to the traditional GDM process exposed in subsection II-A the following modules:
- Delegation module: The delegation module [8] tries to deal with the problems associated to a high number of experts communicating in Web 2.0. Because it is probable that the high number of experts do not participate during the whole GDM process, the delegation module allows an expert to abandon the GDM process leaving the weight of the decision in another participating expert he/she considers similar to. In such a way, an expert is able to select a representative expert for each point of view who will be in charge of defending them. Consequently, the number of experts can be dramatically reduced. The delegation module presents the following steps:

1) The system provides to each expert a ranking containing the $\delta$ experts whose opinions are close to his/her own ones. In the case they have to leave the GDM process, it is possible for them to delegate in those experts since they have similar opinions. Distance for experts $e_{h}$ and $e_{g}$ can be computed using the following expression:

$$
d^{h g}=d^{g h}=\sum_{i=1}^{n} \sum_{j=1, j \neq i}^{n}\left|p_{i j}^{h}-p_{i j}^{g}\right|
$$

where $|\cdot|$ is the absolute value operator and $p_{i j}^{h}$ represents the preferences for expert $e^{h}$ for the alternative $x_{i}$ over $x_{j}$.

2) The expert who has decided to delegate, $e^{h}$, provides a set of trust evaluations, $t_{j}^{h}, j \in\{1, \ldots, m\}$, on the remaining experts. The linguistic term set $T S=\left\{t s_{-3}, t s_{-2}, t s_{-1}, t s_{0}, t s_{1}, t s_{2}, t s_{3}\right\}$ can be used for this purpose. Negative indexes indicate negative evaluations while positive ones indicate that the expert is trusted. Because it is not possible for an expert to provide trust values for all the experts participating in the process, the system will assign the neutral value, $t s_{0}$, to the experts that have not being mentioned by the one that is delegating.

3) In each GDM round, for every expert who has provided a trust evaluation $t_{j}^{h}$, value $t t^{h}=\sum_{j=1}^{m}\left|t_{j}^{h}\right|$ is computed being $\left|t_{j}^{h}\right|$ the index of the provided label. The importance of each expert $e^{j}$ in the GDM process, $\tau^{j}$, can be updated using trust values with the following expression: $\tau^{j}=\tau^{j}+\Delta \tau^{j}$ where $\Delta \tau^{j}=\tau^{h} \cdot\left(t_{j}^{h} / t t^{h}\right)$. An expert who has delegated is provided with a weight value of $0, \tau^{h}=0$ because he/she is not participating.

- Alternatives set selection module: As it has been commented in the introduction, Web 2.0 technologies have promoted an exponential increase on the information available on the Internet. When carrying out debates about that information, it is easy for the experts to get lost due to the high number of possibilities. In order to solve this problem, Fuzzy Ontologies are used. They can help experts to focus on the discussion only of those alternatives that are worth of consideration. In such a way, it is possible to reduce the huge available set of 
alternatives into a feasible one using a set of desirable characteristics [23]. For example, if a set of experts have to choose a company in which to invest money, it would be desirable for them to choose only from companies that would generate profit instead of discussing all the possible companies available in the market. Since there are parameters that determine if a company will provide a profit, a Fuzzy Ontology using that information can be built to determine a reduced list of alternatives companies. A Fuzzy Ontology for storing a certain kind of information can be built using the following steps:

1) Finding and preprocessing data: First, all the information wished to be stored in the Fuzzy Ontology must be collated. For this purpose, it is important to select the information sources carefully. The better the quality of the information, the more reliable will be the Fuzzy Ontology. If information is expressed using different representation methods, it is mandatory to carry out an unification process. It is important to notice that all the information referring to the same concepts must be expressed uniformly. In the case of linguistic values, multigranular fuzzy linguistic modellings can be used for this purpose [24]. The preprocessing process is a critical step since it can influence dramatically the query efficiency. The more refined the data is, the less time it will be consumed carrying out data transformations in the ontology searching step.

2) Query design: This part deals with the definition of the way users will use to communicate with the Fuzzy Ontology in order to retrieve the requested information. Query design step is closely linked to how the data is represented in the Fuzzy Ontology. That is why it is important to represent the data in such a way that the least possible computations are carried out by the system.

3) Validation: After ontology creation, the ontology must be validated in order to check that it works correctly and the results obtained are the expected ones. In [25], authors present several ways of validating ontologies.

4) Storing the Fuzzy Ontology on the Internet: Once the Fuzzy Ontology has been designed, an implementation must be carried out. In order to take advantage of Web 2.0 technologies, the Fuzzy Ontology can be placed onto a server that can manage the Internet users requests and provide them with the required information. For an effective performance, the following elements must be present in the server (See Figure 3):

- Information storage unit: The information storage unit is in charge of storing all the information that conform the ontology. Information must be sorted in order for the ontology reasoner to efficiently carry out queries over it.

- Users requests manager: The users request man-

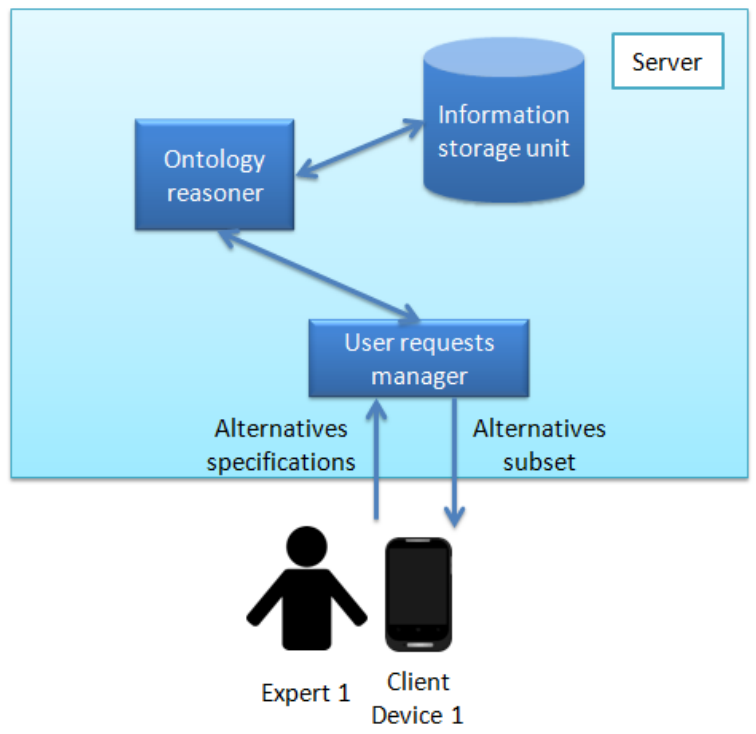

Fig. 3. Fuzzy Ontology query processing scheme.

ager is a module that is in charge of collecting the users queries. Each query is sent to the ontology reasoner in order to calculate the required results.

- Ontology reasoner: The ontology reasoner is in charge of retrieving from the Fuzzy Ontology the information requested by the user in his/her query. The ontology reasoner receives the user query from the users request manager and returns the requested information to it.

The reduced set of alternatives for a GDM process can be computed using the following steps:

1) User connection: The GDM moderator tries to connect to the Fuzzy Ontology server.

2) Query providing: If the previous step is fulfilled and the server accepts the connection, the moderator introduces a set of specifications that the desired alternatives must fulfil.

3) Ontology searching: The ontology reasoner receives the query from the moderator and, using the Fuzzy Ontology information stored in the server, the query is resolved and results are sent back to the users request manager.

4) Results presentation: The reduced set of alternatives received is the one used for the GDM process. They are sent in a user-friendly manner to the client devices in order for users to consult them.

\section{B. Operation way of the proposed GDM process}

The novel designed GDM process that works in Web 2.0 environments follows these steps:

1) Defining the GDM process: First, the GDM process is created over the server. For that purpose, one of the users known as the manager introduces the GDM process data in order to create an instance in the server. Data 
about GDM process purpose and information about the involved users is needed.

2) Alternatives set calculation: Using the alternatives set selection module, the set of alternatives that the experts will discuss is generated.

3) Users provide their preferences: Once that the GDM process is created, users can connect to it with their client devices and provide their preferences. Information is dealt with by the preference dealing module that stores it in a database for posterior computations. In a close GDM process, a username and a password is required in order to grant access only to the involved users. The users registration module is in charge of this task. Once that the preferences are provided, they are aggregated giving to each expert preferences the weight specified by his/her trust value.

4) Calculation of consensus measures: Once that the providing step is ended, consensus measures are calculated. Thanks to them, the system can estimate the agreement among the experts. In the case of a low level agreement, information about the most controversial discussion points can be shown to the users in order for them to focus their debate on them. When the consensus is high, no further discussion is needed and a final ranking of the alternatives can be produced.

5) Feedback calculation: Alongside with consensus measures calculation, it is possible to generate suggestions in order to assist users about how to modify their preferences to increase consensus. This way, the users can notice which preferences values they would have to relinquish in order to increase the overall consensus. It is important to point out that the final decision regarding the modification of the preferences rests on the users, the system only provide recommendations.

6) Further discussion and preferences modification: When consensus is not reached, the debate must keep going. Thanks to the consensus and feedback information, experts can continue their debate in an organized way, focusing in those matters where there is disagreement. At this point, experts can change their minds and modify their preferences. The server can refresh the consensus and feedback information from time to time in order for users to manage up-to-date data. Experts can leave the GDM for a while and delegate into other experts using the delegation module.

7) Calculation of final decision results: If the consensus is high or the specified period of time/number of rounds to make the decision has been reached, the GDM process ends. Final ranking alternative is showed to the users. The GDM process presentation module is in charge of formatting the information stored in the database and present it to the users via their device clients.

A scheme of this architecture and process is shown in Figure 4. It is worth noting that an architecture client-server has been chosen for design purposes. There is a main server that store all the information and users located anywhere in the world

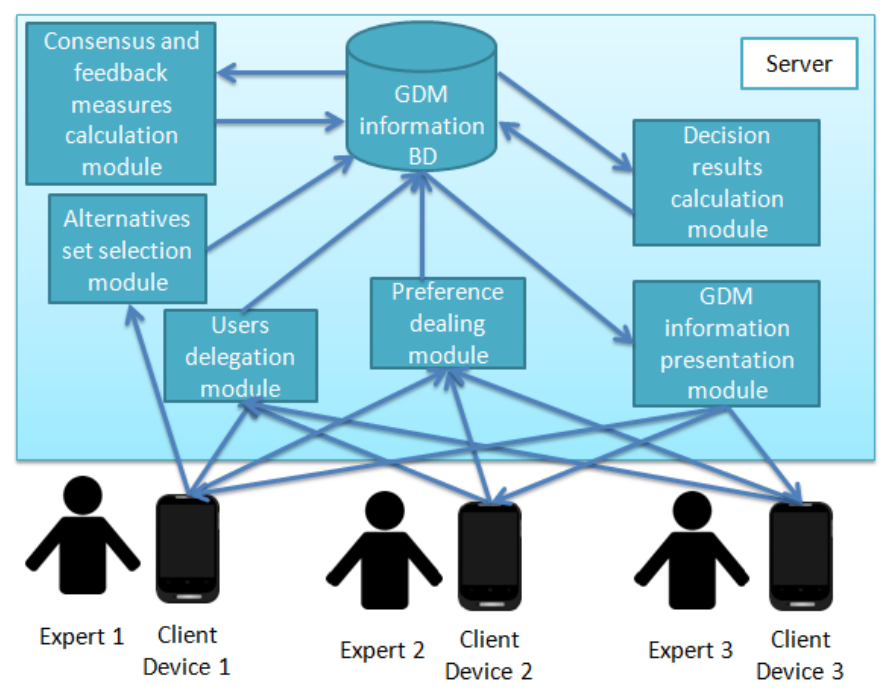

Fig. 4. GDM process implemented over the Internet.

can connect to it using their personal client devices.

\section{Discussion}

In this contribution, a novel GDM method for making decisions in a Web 2.0 environment has been presented. This method allows users from all over the world to gather in Internet communities to make decisions together. Thanks to Fuzzy Ontologies, the method is able to categorize the large amount of information available on the Web making it easy for experts to focus the discussion on the alternatives that fulfil a set of desirable characteristic, such a priori profit. Thanks to the delegation module, experts can abandon the decision process at any time giving their support to a subset of experts.

In this approach, a client-server architecture has been proposed. This architecture has several advantages and drawbacks. One of the main advantages is that client devices do not have to store any information within them in order to work. This way, all the computations are carried out in servers that can be located anywhere. Since huge servers have better computation capability than, for example, smartphones, this way of distributing computations increase the system efficiency. Nevertheless, because all the information is stored in one place, there is a maximum number of requests that the server can support. If a huge amount of users access the system information at the same time, the server can collapse making the information impossible to access. One way of addressing this issue is to use a distributed architecture [26]. In such a way, the information is split and stored in different servers. Therefore, each query resolving unit must request to other servers the required information to carry out computations. The advantage of this system architecture is that, if one server fails, the rest of the information is still available and most of the computations can go on as normal. Its main disadvantage is that is less computational efficient than the client server architecture since more operations must be carried out.

Thanks to Web 2.0 technologies and GDM methods, users 
can communicate, debate and make decisions without having to meet together. Consensus and feedback measures allow users to know when an agreement has been reached and when more debate is needed. Thanks to it, there is no need to extend debates when it is unnecessary and decision are not made in a rush. Another advantage of using GDM methods over Web 2.0 technologies is that they allow people to make decisions in an organized way even when they are far away and can only communicate through their personal Internet devices. Also, they do not have to assist the whole GDM process since they can delegate the decision in their trusted users.

Due to the high amount of information that all the Internet users generate, sometimes it is difficult to distinguish between useful and useless information. Thanks to Fuzzy Ontologies, it is possible to sort, in an organized way, the high amount of information available making it easier for users to manage it. This way, Fuzzy Ontologies can be used in order to focus the GDM discussion in those alternatives that are relevant. Consequently, the GDM complexity is reduced due to the fact that discussion is carried out only on a feasible set of alternatives.

\section{Conclusion}

Web 2.0 technologies and smartphones have revolutionized the way Internet was conceived. Nowadays, Web 2.0 technologies allow millions of users located anywhere in the world to communicate and share information. Smartphones allow users to access the Internet anywhere, without having to go to an Internet access point. The extreme increase in users and information that involves the new Internet paradigm has promoted the necessity of tools that are capable of managing all the information in an organized way. Tools that help a high number of users to cooperate are also needed.

In this contribution, a novel GDM method is presented in order to solve some of the challenges that the use of Web 2.0 technologies entails. GDM methods help users located anywhere to keep in contact, carry out debates and make decisions together, even if they are a high number of users. Fuzzy Ontologies can be used to sort the information. This way, irrelevant information is discarded from the discussion.

\section{ACKNOWLEDGMENT}

This paper has been developed with the financing of FEDER funds in TIN2013-40658-P and Andalusian Excellence Project TIC-5991.

\section{REFERENCES}

[1] P. Andersen, What is Web 2.0?: ideas, technologies and implications for education (Vol. 1, No. 1). Bristol, UK: JISC, 2007.

[2] W. Lehr and L. W. Mcknight, "Wireless internet access: 3G vs. WiFi?!", Telecommunications Policy, Vol. 27, No. 5, pp. 351-370, 2003.

[3] A. Oulasvirta, T. Rattenbury, L. Ma and E. Raita, "Habits make smartphone use more pervasive", Personal and Ubiquitous Computing, Vol. 16, No. 1, pp. 105-114, 2012.

[4] M. Butler, "Android: changing the mobile landscape", Pervasive Computing, IEEE, Vol. 10, No. 1, pp. 4-7, 2011.

[5] D. Mark, J. LaMarche and J. Nutting, Beginning iPhone 4 development: Exploring the IOS SDK, Apress, 2011.
[6] J. M. Blin and A. Satterthwaite, "Individual decisions and group decisions: The fundamental differences", Journal of Public Economics, Vol. 10, No. 2, pp. 247-267, 1978.

[7] A. Lewandowski, "SCDAS - Decision support system for Group Decision Making: Decision Theoretical Framework", Decision Support Systems, Vol. 5, No. 4, pp. 403-423, 1989.

[8] S. Alonso, I. J. Pérez, F. J. Cabrerizo and E. Herrera-Viedma, ”A linguistic consensus model for web 2.0 communities". Applied Soft Computing, Vol. 13, No. 1, pp. 149-157. 2013.

[9] F. Herrera, E. Herrera-Viedma, "Linguistic decision analysis: steps for solving decision problems under linguistic information", Fuzzy Sets and Systems, Vol. 115, No. 1, pp. 67-82, 2000

[10] F. Herrera, S. Alonso, F. Chiclana, E. Herrera-Viedma, "Computing with words in decision making: foundations, trends and prospects", Fuzzy Optimization and Decision Making, Vol. 8 No. 4, pp. 337-364, 2009.

[11] Y. Dong, X. Chen and F. Herrera, "Minimizing adjusted simple terms in the consensus reaching process with hesitant linguistic assessments in Group Decision Making”, Information Sciences, Vol. 297, pp. 95117, 2015.

[12] F.J. Cabrerizo, M.R. Ureña, W. Pedrycz and E. Herrera-Viedma, "Building consensus in Group Decision Making with an allocation of information granularity", Fuzzy Sets and Systems, Vol. 255, pp. 115-127, 2014.

[13] F. Chiclana, F. Herrera and E. Herrera-Viedma, "Integrating three representation models in fuzzy multipurpose decision making based on fuzzy preference relations", Fuzzy Sets and Systems, Vol. 97, No. 1, pp. 33-48, 1998.

[14] I.J. Pérez, F.J. Cabrerizo and E. Herrera-Viedma, "A Mobile Group Decision Making Model for Heterogeneous Information and Changeable Decision Contexts", International Journal of Uncertainty, Fuzziness and Knowledge-Based Systems, Vol. 19, No. 1, pp. 33-52, 2011.

[15] D. Fensel, Ontologies, Springer Berlin Heidelberg, 2001.

[16] T. Lukasiewicz and U. Straccia, "Managing uncertainty and vagueness in description logics for the Semantic Web", Web Semantics: Science, Services and Agents on the World Wide Web, Vol. 6, No. 4, pp. 291-308, 2008.

[17] F. Bobillo and U. Straccia, "Fuzzy ontology representation using OWL 2". International Journal of Approximate Reasoning, Vol. 52, No. 7, pp. 1073-1094, 2011.

[18] F. Baader, D. Calvanese, D. McGuinness, D. Nardi and P. PatelSchneider, The description logic handbook: Theory, implementation and applications, Cambridge University Press, 2003.

[19] S. Calegari and D. Ciucci, "Fuzzy ontology, fuzzy description logics and fuzzy-OWL", Applications of Fuzzy Sets Theory, Vol. 4578, pp. 118-126, 2007.

[20] L. A. Zadeh, "Fuzzy Sets", Information and Control, Vol. 8, No. 3, pp. 338-353, 1965.

[21] N. D. Rodríguez, M. P. Cuéllar, J. Lilius and M. D. Calvo-Flores, "A fuzzy ontology for semantic modelling and recognition of human behaviour", Knowledge-Based Systems, Vol. 66, pp. 46-60, 2014.

[22] A. D. Torshizi, M. H. F. Zarandi, G. D. Torshizi and K. Eghbali, ”A hybrid fuzzy-ontology based intelligent system to determine level of severity and treatment recommendation for Benign Prostatic Hyperplasia", Computer methods and programs in biomedicine, Vol. 113, No. 1, pp. 301-313, 2014.

[23] I.J. Pérez, R. Wikstrom, J. Mezei, C. Carlsson and E. Herrera-Viedma, "A new consensus model for group decision making using fuzzy ontology", Soft Computing, Vol. 17, No. 9, pp. 1617-1627, 2013.

[24] J. A. Morente-Molinera, I. J. Pérez, M. R. Ureña, and E. Herrera-Viedma (2015), "On multi-granular fuzzy linguistic modeling in Group Decision Making problems: A systematic review and future trends", KnowledgeBased Systems, Vol. 74, pp. 49-60, 2015.

[25] P. D. Haghighi, F. Burstein, A. Zaslavsky, and P. Arbon, "Development and evaluation of ontology for intelligent decision support in medical emergency management for mass gatherings", Decision Support Systems, Vol. 54, No. 2, pp. 1192-1204, 2013.

[26] Y. Zhao and X. Qian, "A Distributed Network Architecture Design for ECommerce Sites", Applied Mechanics and Materials, Vol. 543, pp. 30003003, 2014. 